\title{
Learning from Demonstration Using MDP Induced Metrics
}

\author{
Francisco S. Melo ${ }^{1}$ and Manuel Lopes ${ }^{2}$ \\ 1 INESC-ID, TagusPark - Edifício IST \\ 2780-990 Porto Salvo, Portugal \\ fmelo@inesc-id.pt \\ 2 University of Plymouth \\ Plymouth, Devon, PL4 8AA, UK \\ manuel. lopes@plymouth.ac.uk
}

\begin{abstract}
In this paper we address the problem of learning a policy from demonstration. Assuming that the policy to be learned is the optimal policy for an underlying MDP, we propose a novel way of leveraging the underlying MDP structure in a kernel-based approach. Our proposed approach rests on the insight that the MDP structure can be encapsulated into an adequate state-space metric. In particular we show that, using MDP metrics, we are able to cast the problem of learning from demonstration as a classification problem and attain similar generalization performance as methods based on inverse reinforcement learning at a much lower online computational cost. Our method is also able to attain superior generalization than other supervised learning methods that fail to consider the MDP structure.
\end{abstract}

\section{Introduction}

In this paper we address the problem of learning a policy from demonstration. This problem has garnered particular interest in recent years, as the ability of non-technical users to program complex systems to perform customized tasks rests heavily on fast and efficient solutions to this particular problem. The literature on this topic is too extensive to review here, and we refer to $[3,8]$ for reviews.

We formalize the problem of learning a policy from demonstration as a standard supervised learning problem: given a demonstration of some target policy consisting of (possibly perturbed) samples thereof, we must recover the target policy from the observed samples. These samples consist of the actions that the agent (learner) should take at specific situations; the learning agent must generalize the observed actions to situations never observed before.

Several works follow a similar approach to the problem of learning from demonstration. Examples go back to the pioneer work of Pomerleau [13], in which an artificial neural network is used to have a vehicle learn how to steer 
from sample steering motions by a human operator. Other related approaches include $[4,17]$.

There are several interesting aspects in the supervised learning approach. It is a very general formulation of the problem of learning a policy from demonstration, making it possible to leverage the rich body of work on supervised learning. On the other hand, when envisioning real world scenarios - e.g., when a human user must teach an agent some target task - it is seldom practical to have the user provide demonstrations with thousands of samples. This implies that (i) the datasets used will typically be small (say, a couple hundred samples) and amenable to fast learning; and (ii) in large problems, the algorithm must exhibit good generalization ability, and hence all available information on the structure of the problem should be exploited.

Our contribution in this paper addresses the latter issue. Unlike other supervised learning approaches to learning a policy from demonstration, we assume that the target policy is the optimal policy for some underlying Markov decision process (MDP), whose dynamics are known. We thus propose the use of this underlying MDP structure to improve the generalization ability of our learning algorithm. In this sense, our approach is also close to inverse reinforcement learning $[12,14]$ and related approaches (see, e.g., [1] and references therein).

Like IRL-based approaches, our method takes advantage of the underlying MDP structure but avoids one bottleneck associated with the former. In fact, IRL-based methods are typically iterative and require solving one different MDP per iteration, which renders these methods computationally expensive in tasks involving large domains. We instead resort to MDP metrics $[5,22]$ that encapsulate the structure of the MDP and provide a seamless way to leverage it within a supervised learning approach. While many supervised learning methods depend critically on the definition of features that capture useful similarities between elements in input-space, MDP metrics naturally achieve this in the class of problems considered in this paper, by identifying two states as "similar" if policies can safely be generalized from one to the other. ${ }^{3}$

The remainder of the paper is organized as follows. Section 2 formalizes the problem of learning a policy from demonstration. Section 3 reviews IRL-based and supervised learning approaches to this problem. We introduce our method in Section 4 and illustrate its application in Section 5. Section 6 concludes.

\section{Formalizing Learning from Demonstration}

We now formalize the problem of learning a policy from demonstration. Throughout the paper, a policy is understood as a mapping $\pi: \mathcal{X} \rightarrow \Delta(\mathcal{A})$, where $\mathcal{X}$ is some finite set of states, $\mathcal{A}$ is a finite set of actions and $\Delta(\mathcal{A})$ is the set of

\footnotetext{
3 This is particularly evident in scenarios in which the input space is discrete. In fact, problems in continuous domains typically assume the input space as some subset of $\mathbb{R}^{p}$, for which natural metrics exist. The same does not occur in discrete domains, where the notion of two states being close is less immediate.
} 
all probability distributions over $\mathcal{A}$. A policy $\pi$ thus maps each state $x \in \mathcal{X}$ to a distribution over actions. Intuitively, it can be thought of as a decision rule that an agent/decision-maker can follow to choose the actions in each state. All throughout the paper we focus on problems with finite $\mathcal{X}$, typical in scenarios of routing, man-machine interfaces or robot control using high-level skills/options.

A demonstration is a set $\mathcal{D}$ of state-action pairs, $\mathcal{D}=\left\{\left(x_{i}, a_{i}\right), i=1, \ldots, N\right\}$, where the states are independently randomly sampled from $\mathcal{X}$, and the corresponding actions are sampled according to the target policy $\pi_{\text {target }}$. Formally, denoting by Uni $(\cdot)$ the uniform probability distribution over $\mathcal{X}$, we have that the samples $\left(x_{i}, a_{i}\right), i=1, \ldots, N$ are i.i.d. according to

$$
\mathbb{P}[(X, A)=(x, a)]=\operatorname{Uni}(x) \pi_{\text {target }}(x, a),
$$

where $(X, A)=(x, a)$ denotes the event that the pair $(x, a)$ is sampled. ${ }^{4}$

We assume that the target policy $\pi_{\text {target }}$ used to generate the demonstration is either the optimal policy for an underlying Markov decision process (MDP) or a perturbation thereof. A MDP is herein represented as a tuple $(\mathcal{X}, \mathcal{A}, \mathrm{P}, r, \gamma)$, where $\mathcal{X}$ and $\mathcal{A}$ are as defined above, $\mathrm{P}(x, a, y)$ denotes the probability of moving to state $y$ after choosing action $a$ at state $x$ and $r(x)$ is the reward that the agent receives upon reaching state $x$. The constant $\gamma$ is a discount factor. We recall that, given an MDP $\mathcal{M}=(\mathcal{X}, \mathcal{A}, \mathrm{P}, r, \gamma)$, the value associated with a policy $\pi$ in state $x \in \mathcal{X}$ is given by

$$
V^{\pi}(x)=\mathbb{E}_{\pi}\left[\sum_{t=0}^{\infty} \gamma^{t} r\left(X_{t}\right) \mid X_{0}=x\right],
$$

where $X_{t}$ denotes the state of the MDP at time instant $t$ and the expectation $\mathbb{E}_{\pi}[\cdot]$ is taken with respect to the distribution over trajectories of the chain induced by the policy $\pi$. The optimal policy for an MDP is thus the policy $\pi^{*}$ that verifies, for all $x \in \mathcal{X}$ and all $\pi, V^{\pi^{*}}(x) \geq V^{\pi}(x)$. The problem of learning a policy from demonstration can thus be roughly described as that of determining a policy that best approximates the target policy, $\pi_{\text {target }}$, in some sense.

In our finite action setting, we can treat each action $a \in \mathcal{A}$ as a class label, and a policy is essentially a discriminant function that assigns class labels to the states in $\mathcal{X}$. In other words, the policy $\hat{\pi}$ computed by our learning algorithm can be interpreted as classifier that assigns each label $a \in \mathcal{A}$ to a state $x \in \mathcal{X}$ according to the probabilities $\mathbb{P}[A=a \mid X=x]=\hat{\pi}(x, a)$. We henceforth use the designations "classifier" and "policy" interchangeably. We define the $0-1$ loss function $\ell: \mathcal{A} \times \mathcal{A} \rightarrow\{0,1\}$ as $\ell(a, \hat{a})=1-\delta(a, \hat{a})$, where $\delta(\cdot, \cdot)$ is such that $\delta(a, \hat{a})=1$ if $a=\hat{a}$ and 0 otherwise. Our algorithm must then compute the

\footnotetext{
4 There may be applications in which the above representation may not be the most adequate (e.g., in some robotic applications). However, this discussion is out of the scope of the paper and we instead refer to [3].
} 
classifier $\hat{\pi}$ that minimizes the misclassification rate,

$$
\mathcal{E}=\frac{1}{N} \sum_{i=1}^{N} \mathbb{E}_{\hat{\pi}}\left[\ell\left(a_{i}, a\right)\right]=\frac{1}{N} \sum_{i=1}^{N} \sum_{a \in \mathcal{A}} \ell\left(a_{i}, a\right) \hat{\pi}\left(x_{i}, a\right),
$$

where $\left(x_{i}, a_{i}\right)$ is the $i$ th sample in the dataset $\mathcal{D}$. To this purpose, we resort to a kernel-based learning algorithm from the literature [9].

Our contribution arises from considering a kernel obtained from a metric over $\mathcal{X}$ that is induced by the MDP structure. This MDP metric provides a natural way of "injecting" the MDP structure in the learning algorithm, leading to an improved performance when compared to other metrics that ignore the MDP. To put the work of this paper in context, the following section reviews the main ideas behind IRL-based approaches to the problem of learning a policy from demonstration. Also, it shows the supervised learning method used in our experiments to assess the usefulness of MDP metrics in the setting of this paper.

\section{IRL and Supervised Learning Approaches}

In this section we review some background material on (supervised) learning from demonstration and IRL. As will become apparent from our discussion, the underlying MDP used in IRL approaches is a rich structure that, if used adequately, leads to a significant improvement in estimating the target policy. This observation constitutes the main motivation for the ideas contributed in the paper. The section concludes with a brief revision of MDP metrics.

\subsection{Inverse Reinforcement Learning}

To describe the main ideas behind IRL-based approaches to the problem of learning a policy from demonstration, we introduce some additional concepts and notation on Markov decision processes. Given a policy $\pi$ and an MDP $\mathcal{M}=$ $(\mathcal{X}, \mathcal{A}, \mathrm{P}, r, \gamma)$, we have

$$
V^{\pi}(x)=r(x)+\gamma \sum_{y \in \mathcal{X}} \mathrm{P}_{\pi}(x, y) V^{\pi}(y)
$$

where $\mathrm{P}_{\pi}(x, y)=\sum_{a \in \mathcal{A}} \pi(x, a) \mathrm{P}(x, a, y)$. For the particular case of the optimal policy $\pi^{*}$,

$$
V^{*}(x)=r(x)+\max _{a \in \mathcal{A}} \gamma \sum_{y \in \mathcal{X}} \mathrm{P}(x, a, y) V^{*}(y) .
$$

The $Q$-function associated with policy $\pi$ is defined as

$$
Q^{\pi}(x, a)=r(x)+\gamma \sum_{y \in \mathcal{X}} \mathrm{P}(x, a, y) V^{\pi}(y)
$$


Inverse reinforcement learning (IRL) deals with the inverse problem to that of an MDP. Solving an IRL problem consists in recovering the reward function $r$ given the optimal policy $\pi^{*}$ for an MDP. In other words, given a policy $\pi$ and the model $(\mathcal{X}, \mathcal{A}, \mathrm{P}, \gamma)$, we must determine a reward function $r^{*}$ such that $\pi$ is an optimal policy for the $\operatorname{MDP}\left(\mathcal{X}, \mathcal{A}, \mathrm{P}, r^{*}, \gamma\right)$.

IRL was first formalized in the seminal paper by Ng and Russel [12]. Among other things, this paper characterizes the solution space associated with a target policy $\pi_{\text {target }}$ as being the set of rewards $\mathbf{r}$ verifying

$$
\left(\mathrm{P}_{\pi}-\mathrm{P}_{a}\right)\left(\mathbf{I}-\gamma \mathrm{P}_{\pi}\right)^{-1} \mathbf{r} \succeq \mathbf{0},
$$

where we have denoted by $\mathbf{r}$ the column vector with $x$ th component given by $r(x)$. One interesting aspect of the condition (1) is that, although trivially met by some reward functions, it still provides a restriction on the reward space arising solely from considering the structure of the MDP. In other words, by considering the structure of the MDP it is possible to restrict the rewards that are actually compatible with the provided policy.

The sample-based approach to the problem of learning from demonstration considered in this paper, however, is closest to $[10,14]$. In these works, the demonstration provided to the algorithm consists in perturbed samples from the optimal policy associated with the target reward function. Specifically, in [14], the distribution from which the samples are obtained is used as the likelihood function in a Bayesian setting. The paper then estimates the posterior distribution $\mathbb{P}[\mathbf{r} \mid \mathcal{D}]$ using a variant of the MCMC algorithm. In [10], on the other hand, the authors adopt a gradient approach to recover the reward function that minimizes the empirical mean squared error with respect to the target policy.

For future reference, we review the latter method in more detail. Roughly speaking, the working assumption in [10] is that there is one reward function, $r_{\text {target }}$, that the agent must estimate. Denoting the corresponding optimal $Q$ function by $Q_{\text {target }}^{*}$, the paper assumes the demonstrator will choose an action $a \in \mathcal{A}$ in state $x \in \mathcal{X}$ with probability

$$
\mathbb{P}\left[A=a \mid X=x, r_{\text {target }}\right]=\frac{e^{\eta Q_{\text {target }}^{*}(x, a)}}{\sum_{b \in \mathcal{A}} e^{\eta Q_{\text {target }}^{*}(x, b)}},
$$

where $\eta$ is a non-negative constant, henceforth designated as confidence parameter. Now given a demonstration $\mathcal{D}=\left\{\left(x_{i}, a_{i}\right), i=1, \ldots, N\right\}$ obtained according to the distribution in (2), the algorithm proceeds by estimating the reward function $r$ minimizing

$$
\mathcal{E}=\frac{1}{N} \sum_{i}\left(\tilde{\pi}\left(x_{i}, a_{i}\right)-\hat{\pi}_{r}\left(x_{i}, a_{i}\right)\right)^{2}
$$

where $\tilde{\pi}\left(x_{i}, a_{i}\right)$ is the empirical frequency of action $a_{i}$ in state $x_{i}$ and $\hat{\pi}_{r}$ is the optimal policy for the $\operatorname{MDP}(\mathcal{X}, \mathcal{A}, \mathrm{P}, r, \gamma)$. Minimization is achieved by (natural) 
gradient descent, with respect to the parameters of $\hat{\pi}_{\mathbf{r}}-$ the corresponding reward function $\mathbf{r}$. The method thus proceeds by successively updating the reward

$$
r^{(k+1)}=r^{(k)}-\alpha_{t} \tilde{\nabla}_{r} \mathcal{E}\left(r^{(k)}\right),
$$

where $\tilde{\nabla}_{r} \mathcal{E}\left(r^{(k)}\right)$ denotes the natural gradient of $\mathcal{E}$ with respect to $r$ computed at $r^{(k)}$.

We conclude by noting that the computation of the natural gradient $\tilde{\nabla}_{r} \mathcal{E}\left(r^{(k)}\right)$ at each iteration $k$ requires solving the corresponding $\operatorname{MDP}\left(\mathcal{X}, \mathcal{A}, \mathrm{P}, r^{(k)}, \gamma\right)$. This need to solve an MDP at each iteration makes this method computationally expensive for large problems.

\subsection{Supervised Learning}

There is a significant volume of work on supervised learning approaches to the problem of learning a policy from demonstration. That literature is far too extensive to be reviewed here, and we refer to $[3,8]$ for more detailed accounts. Instead, we provide a brief overview of several common traits of all these approaches, and then describe the particular learning algorithm used in Section 5.

First of all, supervised learning approaches typically do not suffer from the inconvenience of having to solve multiple MDPs. This is an appealing property of this class of methods that allows tackling larger problems with significant savings in terms of computational effort. Moreover, we can leverage the rich body of work on supervised learning to the problem of estimating a policy from a demonstration.

However, even when the target policy is assumed optimal for some MDP, most supervised learning approaches to the problem of learning a policy from demonstration eventually ignore the underlying MDP structure in the learning process (see, for example, [4]). This means that such supervised learning algorithms do not actually require any particular knowledge of the underlying structure of the problem to function. Of course, when such information is available, it can provide valuable information for the learning algorithm and the learning algorithm should be able to accommodate such information.

In Section 4 we propose a principled and seamless approach to leverage the MDP structure in a kernel-based learning algorithm. Our proposed approach rests on the insight that the MDP structure can be encapsulated into an inputspace metric that the learning algorithm can use to generalize along the (implicit) MDP structure. We propose using a metric that "projects" the structure of the MDP in such a way that the target policy in two states that are "close" is likely to be similar. This notion is illustrated in the example of Fig. 1.

We postpone to Section 4 the detailed description of how to encapsulate the MDP structure in the input-space metric and conclude this section by describing the learning algorithm used in our experiments.

Going back to the formulation in Section 2, we are interested in computing a classifier $\hat{\pi}$ (a distribution over the set of class labels - in this case, the action 


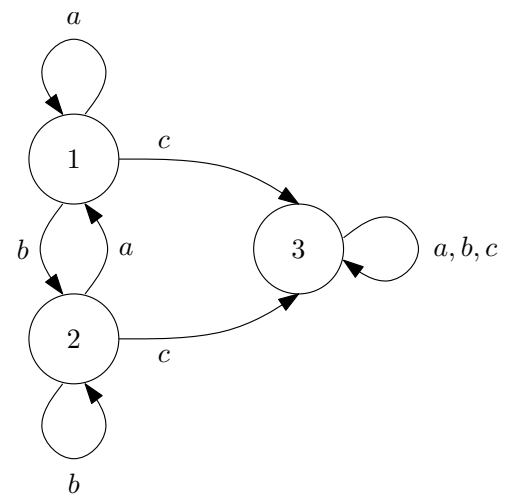

Fig. 1. Transition diagram for a simple MDP with 3 states, 3 actions and deterministic transitions. Even if the reward for this MDP is unknown, the demonstration of an optimal action in state 1 , say action $c$, immediately implies that this action is also optimal in state 2 . In fact, if $c$ is optimal in state 1 , then $r(3)>r(1)$ and $r(3)>r(2)$ - otherwise $c$ would not be optimal in state 1 . But then, the action in state 2 should also be $c$.

set $\mathcal{A})$ that varies along the state-space $\mathcal{X}$. In particular, we are interested in a learning algorithm that takes advantage of the metric structure in $\mathcal{X}$ to be described next. Possible methods for this include kernel logistic regression [23], beta regression [9] and others. We adopt the trivial extension of the latter to the multi-class case due to the its computational efficiency and the possibility of including prior information.

At each state $x \in \mathcal{X}$, the desired classifier $\hat{\pi}$ is simply a multinomial distribution over the set of possible actions. Using a standard Bayesian approach, we compute a posterior distribution over each of the parameters of this multinomial using the corresponding conjugate prior, the Dirichlet distribution. For notational convenience, let us for the moment denote the parameters of the multinomial at a state $x \in \mathcal{X}$ as a vector $\mathbf{p}(x)$ with ath component given by $\mathbf{p}_{a}(x)$. Using this notation, we want to estimate, for each $x \in \mathcal{X}$, the posterior distribution $\mathbb{P}[\mathbf{p}(x) \mid \mathcal{D}]$. Let $x_{i}$ be some state observed in the demonstration $\mathcal{D}$, and let $n_{a}\left(x_{i}\right)$ denote the number of times that, in the demonstration, the action $a$ was observed in state $x_{i}$. Finally, let $n\left(x_{i}\right)=\sum_{a} n_{a}\left(x_{i}\right)$. We have

$$
\begin{aligned}
\mathbb{P}\left[\mathbf{p}\left(x_{i}\right) \mid \mathcal{D}\right] & \propto \operatorname{Multi}\left(\mathbf{p}_{1}\left(x_{i}\right), \mathbf{p}_{2}\left(x_{i}\right), \ldots, \mathbf{p}_{|\mathcal{A}|}\left(x_{i}\right)\right) \operatorname{Dir}\left(\alpha_{1}, \alpha_{2}, \ldots, \alpha_{|\mathcal{A}|}\right) \\
& =\frac{n\left(x_{i}\right) !}{\prod_{a \in \mathcal{A}} n_{a}\left(x_{i}\right) !} \prod_{a \in \mathcal{A}} \mathbf{p}_{a}\left(x_{i}\right)^{n_{a}} \frac{1}{B(\boldsymbol{\alpha})} \prod_{a \in \mathcal{A}} \mathbf{p}_{a}(x)^{\alpha_{a}-1}
\end{aligned}
$$

In other words, the posterior distribution of $\mathbf{p}\left(x_{i}\right)$ is also a Dirichlet distribution with parameters $n_{a}+\alpha_{a}, a=1, \ldots,|\mathcal{A}|$. In order to generalize $\mathbb{P}[\mathbf{p}(x) \mid \mathcal{D}]$ to unvisited states $x \in \mathcal{X}$, and following the approach in [9], we assume that the parameters of the Dirichlet distribution depend smoothly on $x$. From the metric structure of $\mathcal{X}$ we define a kernel on $\mathcal{X}, \mathbf{k}(\cdot, \cdot)$, and use standard kernel regression to extrapolate the parameters of the Dirichlet from the training dataset to unvisited states [18]. Specifically, for any query point $x^{*} \in \mathcal{X}$ and all $a \in \mathcal{A}$, we have

$$
\hat{n}_{a}\left(x^{*}\right)=\sum_{i} \mathbf{k}\left(x^{*}, x_{i}\right) n_{a}\left(x_{i}\right)+\alpha_{a} .
$$


Finally, the posterior mean of the distribution over the parameters $\mathbf{p}\left(x^{*}\right)$ - that we will henceforth use as our classifier at $x^{*}, \hat{\pi}\left(x^{*}, \cdot\right)$ - is given by

$$
\hat{\pi}\left(x^{*}, a\right)=\mathbb{E}\left[\mathbf{p}_{a}\left(x^{*}\right) \mid \mathcal{D}\right]=\frac{\hat{n}_{a}\left(x^{*}\right)}{\sum_{b} \hat{n}_{b}\left(x^{*}\right)},
$$

for all $a \in \mathcal{A}$. In the next section, we introduce our main contribution, proposing a principled way to encapsulate the MDP structure in a suitable metric for $\mathcal{X}$ that can then be used to define the kernel $\mathbf{k}(\cdot, \cdot)$.

We conclude by illustrating the performance of the algorithm above with a simple metric in a problem of learning from demonstration in a grid-like world (a more detailed description of the experimental setting is postponed to Section 5). Figure 2 compares the performance of the algorithm against those of a random policy and a policy obtained by solving the underlying MDP with a random reward. Note that, even using a random reward, the MDP-based solution is able to attain about a policy with $57 \%$ of correct actions, clearly establishing the advantage of using the MDP structure.

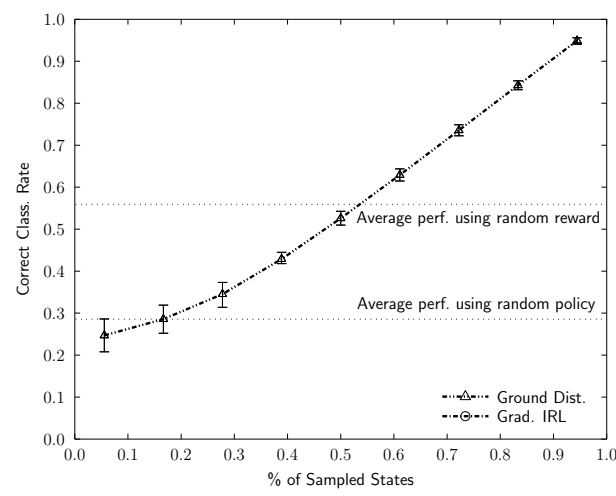

Fig. 2. Classification rate of the method above different demonstration sizes. The horizontal lines correspond to the performances of a random policy and a policy obtained from the underlying MDP with a random reward.

\subsection{Bisimulation and MDP Metrics}

Let us start by considering again the MDP depicted in Fig. 1. In this MDP, there is a close relation between states 1 and 2 since their actions and corresponding transitions are similar. In such a scenario, information about the policy, say, in state 1 will typically be also useful in determining the policy in state 2 .

This notion of "similarity" has recently been explored in the MDP literature as a means to render solution methods for MDPs more efficient $[5,16,22]$. In fact, by identifying "similar" states in an MDP $\mathcal{M}$, it may be possible to construct a smaller MDP $\mathcal{M}^{\prime}$ that can more easily be solved. In this paper we instead use MDP metrics to identify "similar" states and safely generalize the policy observed in the demonstration.

As established in [7], "similarity" between MDP states is best captured by the notion of bisimulation. Bisimulation is an equivalence relation $\sim$ on $\mathcal{X}$ in 
which two states $x$ and $y$ are similar if $r(x)=r(y)$ and

$$
\mathbb{P}\left[X_{t+1} \in U \mid X_{t}=x, A_{t}=a\right]=\mathbb{P}\left[X_{t+1} \in U \mid X_{t}=y, A_{t}=a\right],
$$

where $U$ is some set in the partition induced by $\sim$. Lax bisimulation is an generalization of bisimulation that also accounts for action relabeling. Both bisimulation and lax bisimulation led to the development of several MDP metrics in which, if the distance between two states $x, y$ is zero, then $x \sim y[5,22]$. In this paper we adopt one such MDP metric, introduced in [22] and henceforth denoted as $\delta_{d_{\mathrm{MDP}}}$, that is built over an initial metric on $\mathcal{X}$ that we refer as the ground distance (see Appendix A for details). We point out, however, that this choice is not unique.

While MDP metrics such as the one above were designed to improve efficiency in MDP solution methods, in this paper we are interested in their use in the problem of learning a policy from demonstration. In this context, MDP metrics arise as a natural way to "embed" the MDP structure in a supervised learning algorithm to improve its generalization performance while avoiding solving multiple MDPs. As will soon become apparent from our results, the use of an MDP metric indeed provides a significant and consistent improvement in performance over other metrics that ignore the MDP structure.

\section{A Kernel-based Approach Using MDP Metrics}

We now introduce the main contributions of the paper, namely how to use MDP metrics such as the one discussed above to the particular problem considered in this paper.

The first aspect to consider is that, when learning a policy from demonstration, there is no reward information. While most MDP metrics naturally include a component that is reward-dependent, the particular setting considered here implies that the metric used should not include one such term. Secondly, the metric $\delta_{d_{\mathrm{MDP}}}$ used already implicitly provides the learning algorithm with the necessary information on action relabeling. Therefore, in our algorithm we use the kernel

$$
\mathbf{k}((x, a),(y, b))=\exp \left(-\delta_{d_{\mathrm{MDP}}}((x, a),(y, b)) / \sigma\right),
$$

where $\sigma$ denotes the kernel bandwidth, and (3) and (4) become

$$
\begin{aligned}
\hat{n}_{a}\left(x^{*}\right) & =\sum_{i, b} \mathbf{k}\left(\left(x^{*}, a\right),\left(x_{i}, b\right)\right) n_{b}\left(x_{i}\right)+\alpha_{a}, \\
\hat{\pi}\left(x^{*}, a\right) & =\frac{\hat{n}_{a}\left(x^{*}\right)}{\sum_{b} \hat{n}_{b}\left(x^{*}\right)} .
\end{aligned}
$$

The complete algorithm is summarized in Algorithm 1. 


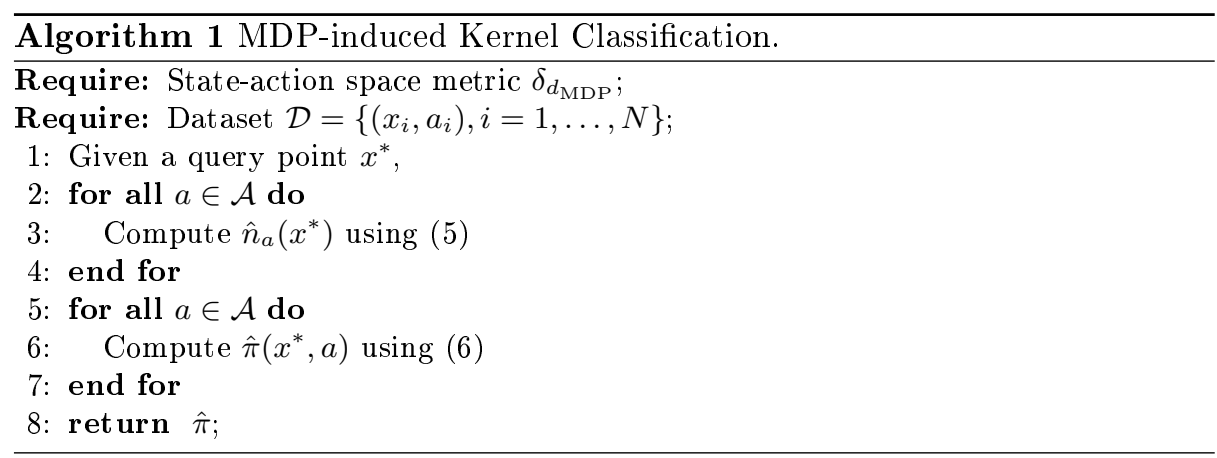

\subsection{Active Learning from Demonstration}

The method described in Algorithm 1 is a Bayesian inference method. This means, in particular, that the algorithms estimates a full posterior distribution over the parameters $\mathbf{p}(x)$ of the policy (see Section 3.2). It is possible to use this posterior distribution in a simple active sampling strategy that may lead to a reduction in the sample complexity of our method [19].

One possibility is to compute the variance associated with the posterior distribution over the parameters $\mathbf{p}(x)$ at each state $x \in \mathcal{X}$, choosing as the next sample the state for which this variance is largest. The intuition behind this idea is that states with larger variance correspond to states that were observed less frequently and/or are distant (in our MDP metric sense) from more sampled states.

Using this active learning strategy we can reduce the number of samples required for learning, since more informative states will be chosen first. Note also that this active learning approach implicitly takes into account the similarity between the states, in a sense treating similar states as one "aggregated" state and effectively requiring less samples.

\section{$5 \quad$ Results}

In this section we discuss several aspects concerning the performance of our proposed approach. Our results feature demonstrations with significantly less samples than the size of the state-space of the underlying MDP, allowing for a clearer evaluation of the generalization ability of the tested methods. Also, the test scenarios used are typically not too large, to allow for an easier interpretation of the results.

Our first test aims at verifying the general applicability of our approach. To this purpose, we applied the algorithm in Section 3.2 using the MDP metric described in Section 4 to 50 randomly generated MDPs. The state-space of the MDPs varies between 20 and 60 states, and the action space between 4 and 10 actions. From each state, it is possible to transition to between $20 \%$ and $40 \%$ of the other states. For each MDP, we randomly sample (without replacement) 
half of the states in the MDP and provide the learning algorithm with a demonstration consisting of these states and the corresponding optimal actions.

We compare the performance of the algorithm in Section 3.2 when using the MDP metric against the performance of that same algorithm when using other metrics. In particular, we used the zero-one metric, in which each the distance between any two state-action pairs $(x, a)$ and $(y, b)$ is either zero - if $(x, a)=(y, b)-$ or 1 , and the transition distance, in which the distance between any two state-action pairs $(x, a)$ and $(y, b)$ corresponds to the minimum number of steps that an agent would take to move from state $x$ to state $y$ when taking as a first action the action $a$. As for the MDP metric, we used the distance $\delta_{d_{\mathrm{MDP}}}$ from the transition distance just described. The bandwidth of the kernel was adjusted experimentally for each metric to optimize the performance. The results are summarized in Table 1.

Table 1. Average performance over 50 random worlds with between 20 and 50 states and 4 and 10 actions.

\begin{tabular}{cc}
\hline & Correct Class. Rate (\%) \\
\hline Zero-one Distance & $57.95 \pm 4.33$ \\
Ground Distance & $60.67 \pm 5.10$ \\
$\delta_{d_{\mathrm{MDP}}}$ & $73.92 \pm 6.98$ \\
\hline
\end{tabular}

As is clear from the results above, our approach clearly outperforms all other approaches, computing the correct action on $73 \%$ of the total number of states. This establishes that, as expected, the use of the MDP metric indeed provides an important boost in the performance of the method.

Our second test aims at comparing the performance of our approach against that of IRL-based algorithm (namely, the algorithm in Section 3.1). We run this comparison in two dimensions. We compare how both methods behave as the size of the demonstration increases and as the noise in the demonstration varies. For this comparison, we used a scenario consisting of eight weakly connected clusters of nine states each, in a total of 72 states. Unlike more standard grid-world scenarios, the weakly connected clusters of states imply that there is a great asymmetry between the states in terms of transitions (unlike what occurs in standard grid-world domains). For this MDP, we considered 5 actions, corresponding to motions in the 4 directions, and the "NoOp" action.

We provided the algorithms with demonstrations of increasing size and evaluated the percentage of correct actions. As before, the demonstration was obtained by sampling (without replacement) a percentage of the total number of states and, for these, providing the corresponding optimal actions according to the target policy. Figure 3(a) compares our approach with the gradient-based IRL approach (GIRL). We also depict the performance of the supervised learning method when using other metrics. 


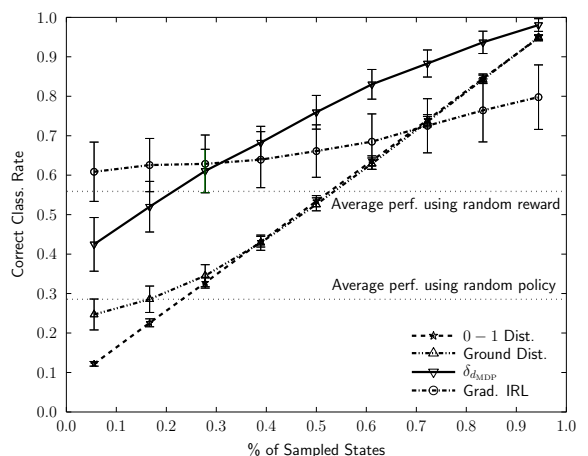

(a)

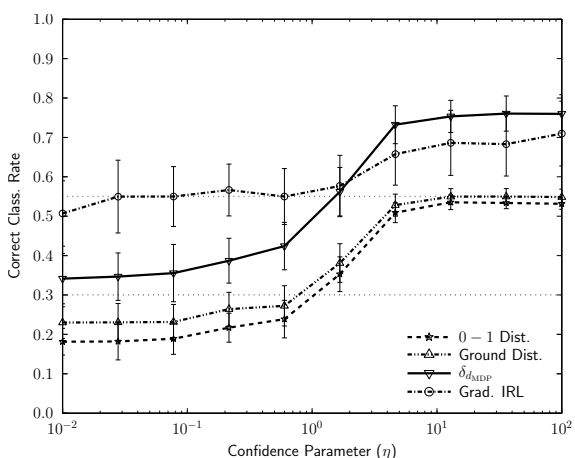

(b)

Fig. 3. Classification rate of the different methods for (a) different demonstration sizes; (b) different confidence levels on the demonstration, with a demonstration size of alf the number of states. The horizontal lines are the baseline references (see main text). The results depicted are averaged over 50 independent Monte-Carlo trials, with the error bars corresponding to sample standard deviation.

For interpretation purposes, we included in the figure two baselines. The lower is obtained by computing the average performance of a random policy $(\approx 30 \%)$. The second baseline is obtained by computing the average performance of the optimal policy obtained from a random reward $(\approx 57 \%)$. It is clear that, because of the inherent MDP structure, the latter baseline is much higher. This further strengthens our point on the use of MDP structure to attain higher generalization.

The results show that all methods improve as the size of the demonstration increases. For very small demonstrations, GIRL gives better results as its baseline is higher. With a dataset larger than about $30 \%$ of the total number of states, our method clearly outperforms all other up to a "full demonstration", when all kernel-based methods perform alike. We also note that the worse performance GIRL even in large demonstrations is probably due to the existence of local minima. ${ }^{5}$

Figure 3(b) compares the performance of all methods as the noise in the demonstration varies. As in the first experiment, we used a demonstration of half the total number of states in the MDP. The actions were sampled from the distribution in (2) and the noise was adjusted by changing the value of the confidence parameter $\eta$. Low values of $\eta$ correspond to noisy policies (where many suboptimal actions are sampled) while high values of $\eta$ correspond to near-optimal policies. As expected all methods behave worse as the noise level increases, approaching the baseline performance levels for high levels of noise. As the noise decreases, the performance approaches the levels observed in Fig. 3(a). We note, however, that the performance of the kernel-based methods suffers a

${ }^{5}$ The results shown contain only runs that did have an improvement from the initial condition. 


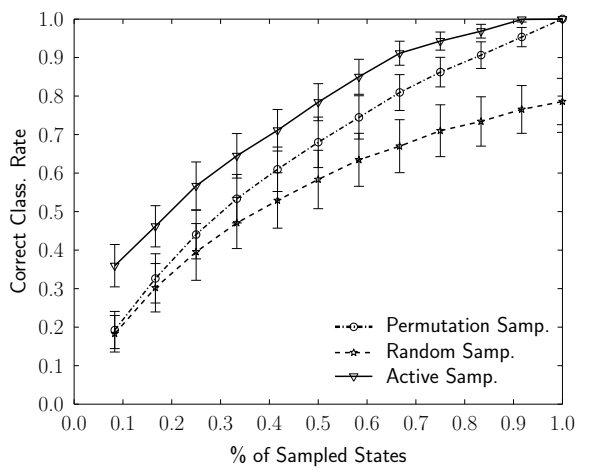

(a)

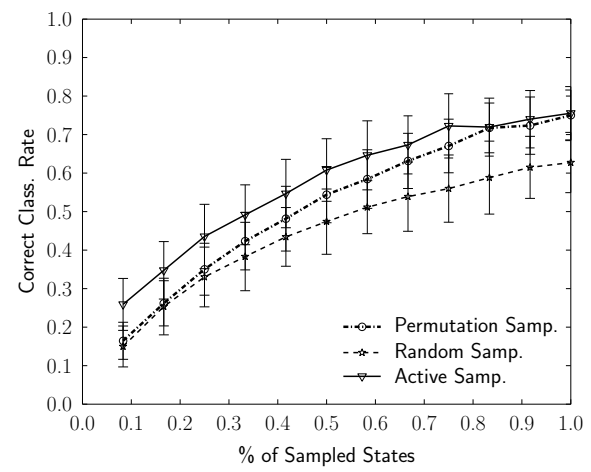

(b)

Fig. 4. Classification rate of the different exploration methods with the size of the demonstration (as a ration of the number of states). Left figure optimal demonstrations, Right figure noisy demonstration

significant improvement at a certain noise threshold (for $\eta \approx 1$ ). We also note that larger demonstrations allowing states to be sampled with replacement will lead all methods to better filter out the noise and improve the classification rate.

Figure 4 shows the results of active learning for our method. We compare several exploration techniques: random exploration - where states are sampled uniformly, biased exploration - where state with less neighbor are chosen more often, normalized sampling - where the sampling is based on a permutation of all the states and our active learning approach based on the variance of the parameters of the posterior distribution.

The figure shows typical results that vary with the problem but have the same relations between the methods. The obtained gain depend on the problem, where problems with higher symmetries and states more clustered will yield and higher gain of the active learning approach. From our results we saw that an active learning approach was never worse than a permutation of states.

\section{Concluding Remarks}

It is clear from our results that the use of the MDP structure indeed provides valuable information when tackling the problem of learning a policy from demonstration. This can be verified easily by comparing, for example, the two baselines in Fig. 2. In these results, using a random policy lead to a correct classification rate of around $30 \%$. However, if we instead use a random reward function and solve the corresponding MDP, the correct classification rate goes up to $57 \%$. This clearly indicates that, in this particular example, the MDP structure significantly restricts the set of possible policies. Although these numbers correspond to a rather small environment in which the set of policies is naturally small, some preliminary experiments indicate that this conclusion holds in general (although with different numbers, of course). This conclusion is also supported by the discussion in Section 3.1 about the results in [12]. 
A second remark is concerned with the computational complexity associated with the particular MDP metric used in our results. As already pointed out by [5], MDP metrics that rely on the Kantorovich metric to evaluate the distance between two distributions $\mathrm{P}(x, a, \cdot)$ and $\mathrm{P}(y, b, \cdot)$ - such as the one used here are computationally demanding. There are other alternative metrics that can be used (such as the total variation distance [5]) that are significantly more computationally efficient and can thus alleviate this issue. Nevertheless, it is worth pointing out that the MDP metric needs only to be computed once and can then be applied to any demonstration. This point is particularly important when envisioning, for example, robotic applications, since the MDP metric can be computed offline, hard-coded into the robot and used to learn different tasks by demonstration to be able to adapt to different users.

Another important point to make is that there are other methods that share a similar principle to the gradient-based IRL method in Section 3.1 and used for comparison in Section 5. These methods, while not explicitly concerned in recovering a reward description of the demonstrated task, use nevertheless an underlying MDP structure within a supervised learning or optimization setting $[2,15,20,21,24]$. Unfortunately, these approaches are close to IRL-based methods in that they still suffer from the need to solve multiple MDPs (see also the discussion in [11] for a more detailed account of the similarities and differences between the methods above). It is also worth noting that most aforementioned methods are designed to run with significantly larger datasets than those used in the experiments [11]. In conclusion they share the same advantages and disadvantages of the GIRL method in Section 3.2

Finally, we conclude by noting that there is no immediate (theoretical) difficulty in extending the ideas in this paper to continuous scenarios. We did not discuss this extension in the paper since MDP metrics in continuous MDPs require a significantly more evolved machinery to describe [6] that would unnecessarily complicate the presentation. On the otherhand, although some continuous MDP metrics have been identified and shown to have good theoretical properties [6], they are expensive to compute and no computationally efficiently alternatives are known. One important avenue for future research is precisely the identifying continuous MDP metrics that are efficiently computable.

\section{A Description of a Lax-Bisimulation Metric}

Let $\mathcal{M}=(\mathcal{X}, \mathcal{A}, \mathrm{P}, r, \gamma)$ be an MDP, where the state-space $\mathcal{X}$ is endowed with a distance $d$. In other words, $(\mathcal{X}, d)$ is a metric space, and we henceforth refer to $d$ as the ground distance. The function $d$ can be any reasonable distance function on $\mathcal{X}$, e.g., the Manhattan distance in the environment of Fig. 1. Given one such distance $d$ and any two distributions $p_{1}$ and $p_{2}$ over $\mathcal{X}$, the Kantorovich distance (also known as the earth 
mover's distance) between $p_{1}$ and $p_{2}$ is defined as the value of the linear program

$$
\begin{array}{llr}
\max _{\theta_{x}} & \sum_{x}\left(p_{1}(x)-p_{2}(x)\right) \theta_{x} & \\
\text { s.t. } & \theta_{x}-\theta_{y} \leq d(x, y), & \text { for all } x, y \in \mathcal{X} \\
& 0 \leq \theta_{x} \leq 1 & \text { for all } x \in \mathcal{X}
\end{array}
$$

and denoted as $\mathrm{K}_{d}\left(p_{1}, p_{2}\right)$. Now given any two state-action pairs $(x, a)$ and $(y, b)$, we write

$$
\delta_{d}((x, a),(y, b))=k_{1}|r(x)-r(y)|+k_{2} \mathrm{~K}_{d}(\mathrm{P}(x, a, \cdot), \mathrm{P}(y, b, \cdot))
$$

where $k_{1}$ and $k_{2}$ are non-negative constants such that $k_{1}+k_{2} \leq 1$. The function $\delta_{d}$ is, in a sense, a "one-step distance" between $(x, a)$ and $(y, b)$. It measures how different $(x, a)$ and $(y, b)$ are in terms of immediate reward/next transition. In order to measure differences in terms of long-term behavior, we denote by $\mathrm{H}_{d}(U, V)$ the Hausdorff distance (associated with $d$ ) between two sets $U$ and $V,{ }^{6}$ and define the MDP metric $d_{\mathrm{MDP}}$ as the fixed point of the operator $\mathbf{F}$ given by

$$
\mathbf{F}(d)(x, y)=\mathrm{H}_{\delta_{d}}(\{x\} \times \mathcal{A},\{y\} \times \mathcal{A}) .
$$

As shown in [22], the metric $d_{\mathrm{MDP}}$ can be obtained by iterating $\mathbf{F}$ and whenever $d(x, y)=0$ then $x$ and $y$ are lax-bisimulation equivalent. Also, using the metric $d_{\mathrm{MDP}}$ it is possible to relabel the actions at each state to match those of "nearby" states.

We conclude by noting that, as discussed in Section 4 , in our results we use $\delta_{d_{\mathrm{MDP}}}$ with $k_{1}=0$ on the right-hand side of (7).

\section{References}

1. Abbeel, P.: Apprenticeship learning and reinforcement learning with application to robotic control. Ph.D. thesis, Dep. Computer Science, Stanford Univ. (2008)

2. Abbeel, P., Ng, A.: Apprenticeship learning via inverse reinforcement learning. In: Proc. 21st Int. Conf. Machine Learning. pp. 1-8 (2004)

3. Argall, B., Chernova, S., Veloso, M.: A survey of robot learning from demonstration. Robotics and Autonomous Systems 57(5), 469-483 (2009)

4. Chernova, S., Veloso, M.: Interactive policy learning through confidence-based autonomy. J. Artificial Intelligence Research 34, 1-25 (2009)

5. Ferns, N., Panangaden, P., Precup, D.: Metrics for finite Markov decision processes. In: Proc. 20th Conf. Uncertainty in Artificial Intelligence. pp. 162-169 (2004)

6. Ferns, N., Panangaden, P., Precup, D.: Metrics for Markov decision processes with infinite state-spaces. In: Proc. 21st Conf. Uncertainty in Artificial Intelligence. pp. 201-208 (2005)

7. Givan, R., Dean, T., Greig, M.: Equivalence notions and model minimization in Markov Decision Processes. Artificial Intelligence 147, 163-223 (2003)

${ }^{6}$ Given a metric space $(\mathcal{X}, d)$, the Hausdorff distance between two sets $U, V \subset \mathcal{X}$ is given by

$$
\mathrm{H}_{d}(U, V)=\max \left\{\sup _{x \in U} \inf _{y \in V} d(x, y), \sup _{y \in V} \inf _{x \in U} d(x, y)\right\} \text {. }
$$


8. Lopes, M., Melo, F., Montesano, L., Santos-Victor, J.: Abstraction levels for robotic imitation: Overview and computational approaches, In: : From Motor Learning to Interaction Learning in Robots, pp. 313-355 (2010)

9. Montesano, L., Lopes, M.: Learning grasping affordances from local visual descriptors. In: Proc. 8th Int. Conf. Development and Learning. pp. 1-6 (2009)

10. Neu, G., Szepesvári, C.: Apprenticeship learning using inverse reinforcement learning and gradient methods. In: Proc. 23rd Conf. Uncertainty in Artificial Intelligence. pp. 295-302 (2007)

11. Neu, G., Szepesvári, C.: Training parsers by inverse reinforcement learning. Machine Learning (2009 (accepted))

12. Ng, A., Russel, S.: Algorithms for inverse reinforcement learning. In: Proc. 17th Int. Conf. Machine Learning. pp. 663-670 (2000)

13. Pomerleau, D.: Efficient training of artificial neural networks for autonomous navigation. Neural Computation 3(1), 88-97 (1991)

14. Ramachandran, D., Amir, E.: Bayesian inverse reinforcement learning. In: Proc. 20th Int. Joint Conf. Artificial Intelligence. pp. 2586-2591 (2007)

15. Ratliff, N., Bagnell, J., Zinkevich, M.: Maximum margin planning. In: Proc. 23rd Int. Conf. Machine Learning. pp. 729-736 (2006)

16. Ravindran, B., Barto, A.: Approximate homomorphisms: A framework for nonexact minimization in Markov decision processes. In: Proc. 5th Int. Conf. Knowledge-Based Computer Systems (2004)

17. Saunders, J., Nehaniv, C., Dautenhahn, K.: Teaching robots by moulding behavior and scaffolding the environment. In: Proc. 1st Annual Conf. Human-Robot Interaction (2006)

18. Schölkopf, B., Smola, A.: Learning with kernels: Support vector machines, regularization, optimization and beyond. MIT Press (2002)

19. Settles, B.: Active learning literature survey. Tech. Rep. CS Tech. Rep. 1648, Univ. Wisconsin-Maddison (2009)

20. Syed, U., Schapire, R.: A game-theoretic approach to apprenticeship learning. In: Adv. Neural Information Proc. Systems. vol. 20, pp. 1449-1456 (2008)

21. Syed, U., Schapire, R., Bowling, M.: Apprenticeship learning using linear programming. In: Proc. 25th Int. Conf. Machine Learning. pp. 1032-1039 (2008)

22. Taylor, J., Precup, D., Panangaden, P.: Bounding performance loss in approximate MDP homomorphisms. In: Adv. Neural Information Proc. Systems. pp. 1649-1656 (2008)

23. Zhu, J., Hastie, T.: Kernel logistic regression and the import vector machine. In: Adv. Neural Information Proc. Systems. pp. 1081-1088 (2002)

24. Ziebart, B., Maas, A., Bagnell, J., Dey, A.: Maximum entropy inverse reinforcement learning. In: Proc. 23rd AAAI Conf. Artificial Intelligence. pp. 1433-1438 (2008) 